\title{
Design of Piezoelectric Tile for Energy Harvesting: Experimental Approach
}

\author{
Ehsan Maani Miandoab ${ }^{1}$, Amir Hossein Jafari², Aref Valipour ${ }^{3}$ \\ 1. School of Engineering Science, College of Engineering, University of Tehran, Iran \\ 2. School of Metallurgy and Materials Engineering, College of Engineering, University of Tehran, Iran \\ 3. School of Electrical \& Computer Engineering, College of Engineering, University of Tehran, Iran \\ E-mail: e.maani@ut.ac.ir; amirhosseinjafari@ut.ac.ir; aref.valipour@ut.ac.ir
}

Received: 29 June 2021; Accepted: 6 August 2021; Available online: 10 October 2021

\begin{abstract}
The generation of electricity by renewable energies is an important need of today's society. Piezoelectric energy harvesting is one of these useful technologies which can generate electricity by applying external force on piezoelectric material. This study illustrates more power generation from piezoelectric tile by changing the situation of piezo discs and connect to proportional electrical circuit. Two different designs of piezoelectric tile are presented by performing experimental analyses. The experimental results showed that placing piezoelectric elements in a bending position leads to higher power generation in comparison with traditional flat positioning, which was approximately 78 times far superior. It is also revealed that by design of an electrical circuit, the tile can be advantageous for lighting in crowded sidewalks with required lighting time. The results of this paper can be beneficial in the design and fabrication of these tiles for different applications.
\end{abstract}

Keywords: Piezoelectric; Smart material; Tile; Optimal design; lighting; Walking energy.

\section{Introduction}

Renewable green energy production recently is noteworthy due to the negative environmental effect of global warming caused by the consumption of fossil fuels. Developing a sustainable source of energy and electricity capable of resolving environmental issues is extremely important, and increasing the use of renewable energy is considered useful and essential [1]. Energy harvesting is one of the most supportive technologies in response to the global energy problems without depleted natural resources. Energy harvesting technology can generate beneficial energy from ineffectual energy sources [2]. The implication of energy harvesting is generating electrical energy from wasted energy such as light [3], heat [4], mechanical deformation, human movement and vibration [5]. Diverse procedures are available for converting mechanical vibration energy into electrical energy, such as electromagnetic, triboelectric and piezoelectric [6]. Newly presented reports in the field of materials have shown considerable progress in self-powered energy sources. Particularly, the usage of piezoelectric materials in the energy harvesters has attracted attention as a simple solution. Piezoelectric energy harvesting technology can be used as self-power in non-battery devices, wireless sensors, etc [7]. Piezoelectric materials have special characteristics that could be able to use as a power source in a piezoelectric energy harvesting system. Broadly, piezoelectric materials are known as anisotropic dielectrics that some of them have ferroelectric or nonferroelectric properties. When placed beneath the mechanical stress, some changes in the atomic structure of the crystal occur. According to these changes, ions in the structure separating and a dipole moment is formed. Thus, it can convert the stress to electricity by this feature. Piezoelectric materials can also make displacement by applying the electric field [8]. Piezoelectric properties exist in many materials such as PZT, Barium Titanate, and PVDF which are the most well-known [9]. The application of piezoelectric in piezoelectric energy harvesting has been increasing. It also will make an enormous market in the foreseeable future. This approach of energy harvesting exists in many concepts such as sidewalk power generation $[10,11]$ or other hectic areas like highways or speed breakers [12] to generate the power from converting kinetic ene11rgy to electricity and harvest the generated power to rechargeable battery and capacitor. This paper is called the design and construction of piezoelectric energy harvesting tile, which measures the energy of the tile by changing some of its mechanical parameters and electronic circuits, and compares the effect of changing the parameters on the final power generated in an experimentally manner.

\section{Optimal design of power generation tile}


Because of electromechanical coupling, piezoelectric materials can convert applied mechanical stress to electrical charge. This characteristic can be used in sensing and energy harvesting applications. The governing equation of electromechanical coupling can be written as Eq. (1).

$$
\{D\}=[e]^{T}\{S\}+\left[\alpha^{S}\right]\{E\}
$$

where $D,[e]^{T},\{S\},\left[\alpha^{S}\right]$ and $\{E\}$ are electric displacement vector, dielectric permittivity matrix, strain vector, dielectric matrix and the electric field vector respectively. In this study, piezoelectric disks are utilized which is shown in Fig. 1a.

(a)

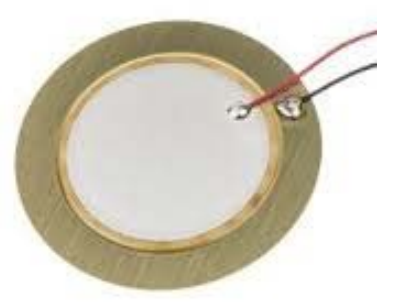

(b)

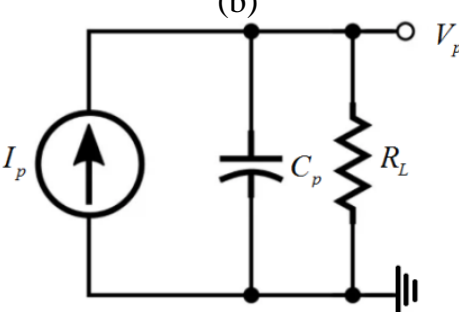

Fig. 1. (a) Diaphragm piezoelectric, (b) Equivalent circuit of piezoelectric

In recently researches the piezoelectric was shown liked as an alternating current source with a parallel capacitor. The equivalent circuit of piezoelectric in energy generation applications is shown in Fig. 1b where $I_{p}$ is the generated current, $C_{P}$ is the internal capacity and $R_{L}$ is the applied load to the piezoelectric. In many previous researches on power generation tiles, piezoelectric disks are located horizontally on the tile base as shown in Fig. 2.

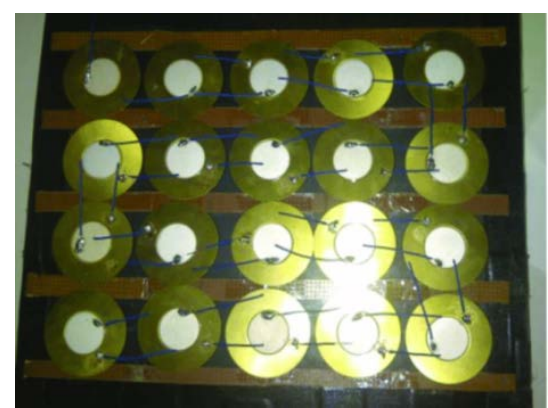

Fig. 2. Piezoelectric disks arrangements on power generation tile [13]

To find an optimal arrangement for piezoelectric disks, Eq. (1) is rewritten as Eq. (2) by noting that there is not any external electric field $(E=0)$.

$$
\{D\}=[e]^{T}\{S\}
$$

It is clear from Eq. (2), that generated electrical displacement is directly dependent to piezo-disk stress. It is expected that titling disks increases their mechanical stress and leads to higher power generation. Thus two tiles with conventional (Tile A) and proposed (Tile B) arrangements are designed and fabricated to analyze the effect of disk placement on tile efficiency which are shown in Fig. 3 .

(a)

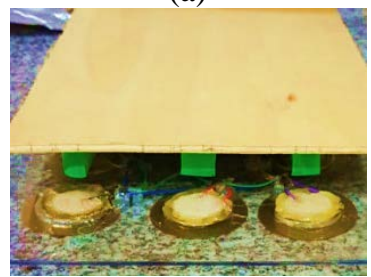

(b)

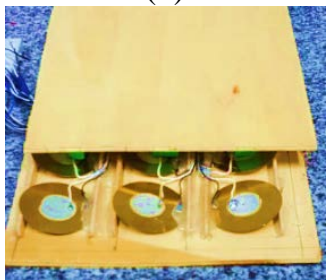

Fig. 3. Piezoelectric tiles (a) Tile 1 (b) Tile 2 
Fig. 4 shows the schematic of system electrical integration. As shown in this figure, nine disks are used in each tile in parallel mode and H-bridge circuits are utilized to make currents in same direction.

To obtaining data from tile, input and output voltages of load resistance $\left(R_{L}\right)$ with the presented schematic in Fig. 5 are monitored using computer and data analyzer.

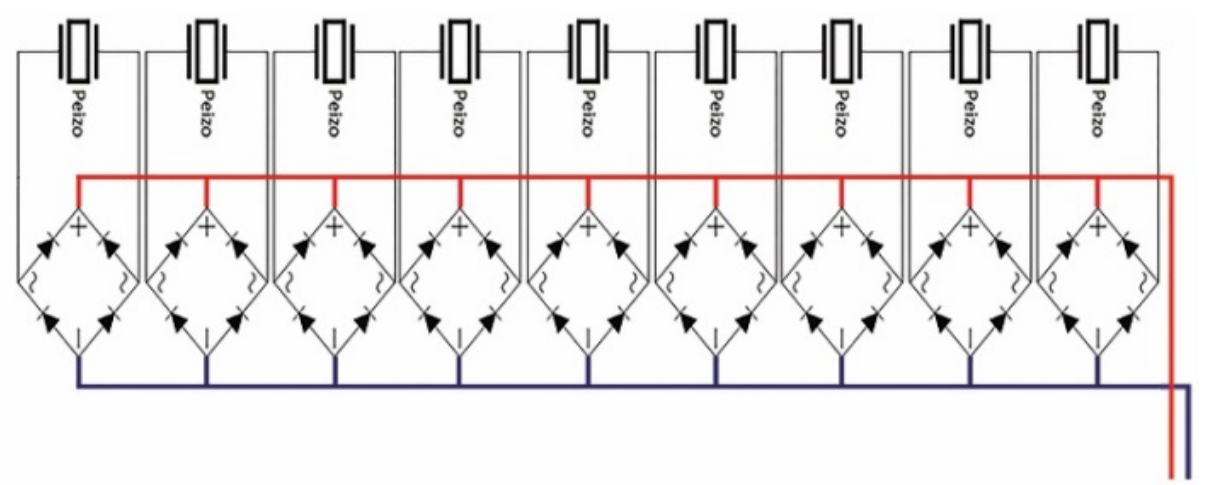

Fig. 4. Electrical integration of piezo-disks in tiles

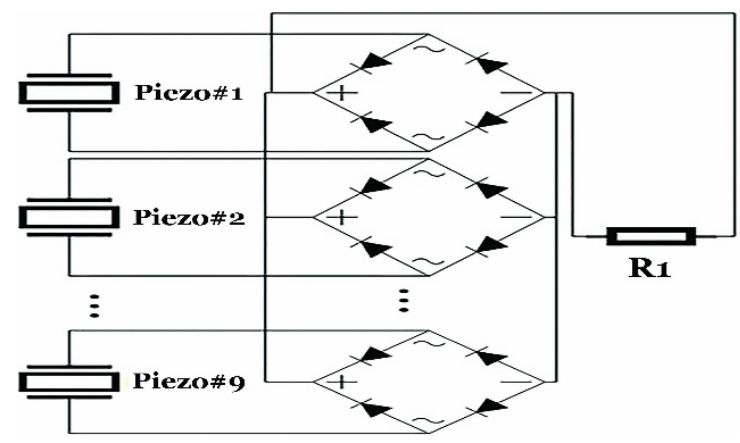

Fig. 5. Electrical integration of piezo-disks in tiles

(a) Tile 1

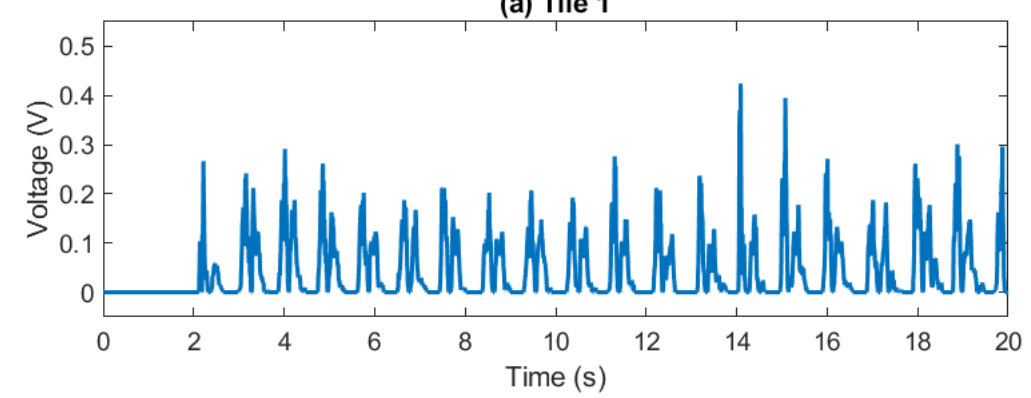

(b) Tile 2

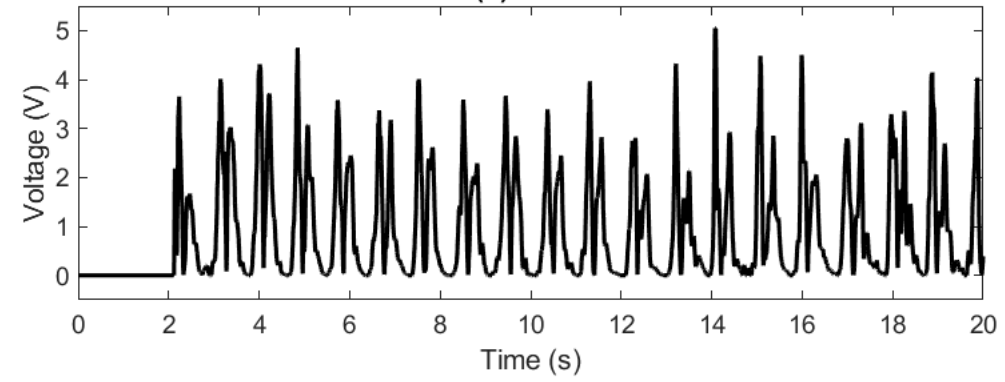

Fig. 6. Output voltages of (a) Tile 1, (b) Tile 2

To show the effect of piezo-disks placement and compare the efficiency of two presented tiles in Fig. 3 in energy harvesting, same dynamic loads are applied to both of them and output voltages are plotted in Fig.6. As 
shown in this figure, the output voltage of modified version, Tile 2, is about 10 times greater than Tile 1 . According to compare these two plots, the explanation for the large difference between the output voltages of the two tile samples is that due to them not being the straight situation of piezo discs in tiles 2, the elements inside tile 2 can move vertically and bend. There are also under more strain than the smooth condition of tile 1 and as a result Given the relationship between strain and the energy produced by the piezo, more voltage can be expected from it. So to produce more voltage and energy, tile design 2 is much preferable.

The generated energy of tile increases by increasing the output voltage of tile. Fig. 7 shows the harvested energy by tiles in 20 sec which reveals that generated energy by Tile 2 is much higher than Tile 1 energy.

As depicted in Fig. 7 and Table 1, tile2 is more sufficient. Hence, by planting tiles over a wider area; they can be used as macro-power sources of energy.

(a) Tile 1

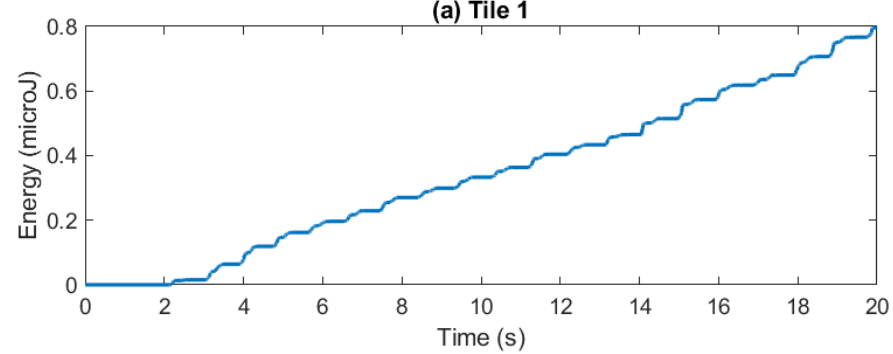

(b) Tile 2

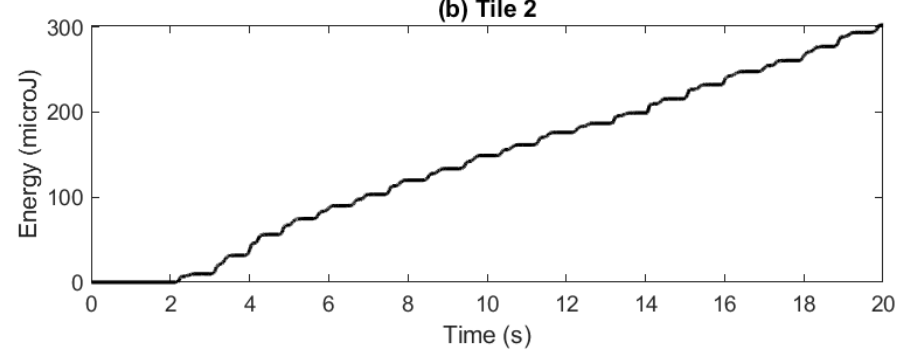

Fig. 7. Generated energy by (a) Tile 1, (b) Tile 2

Table 1. Voltage and energy data obtained from two types of tiles

\begin{tabular}{cccc}
\hline & Output voltage RMS & Output voltage MAX & Max Energy Reached In 20S \\
\hline TILE 1 & $0.0751 \mathrm{~V}$ & $0.4245 \mathrm{~V}$ & $0.7962 \mu \mathrm{J}$ \\
TILE 2 & $1.4290 \mathrm{~V}$ & $5.0500 \mathrm{~V}$ & $302.298 \mu \mathrm{J}$ \\
\hline
\end{tabular}

\section{Design of electrical circuit for lighting Aim}

One of the best applications of power generation tiles is online consumption of generated energy for lighting aim in sidewalks, subways also turning on traffic signs in roads by crossing vehicles. For example, they can be utilized for turning on road light or caution sign by approaching a vehicle to road bending. Fig. 8 shows the designed setup and its schematic for analyzing its performance.

(a)

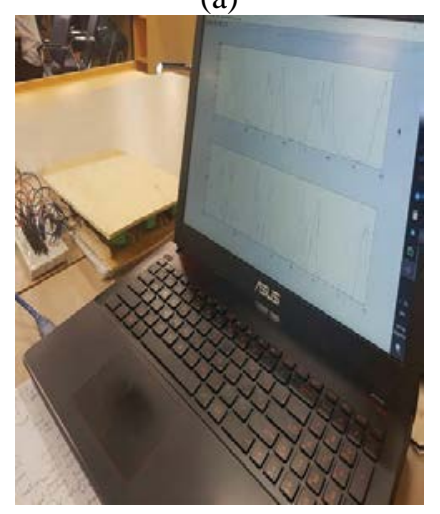

(b)

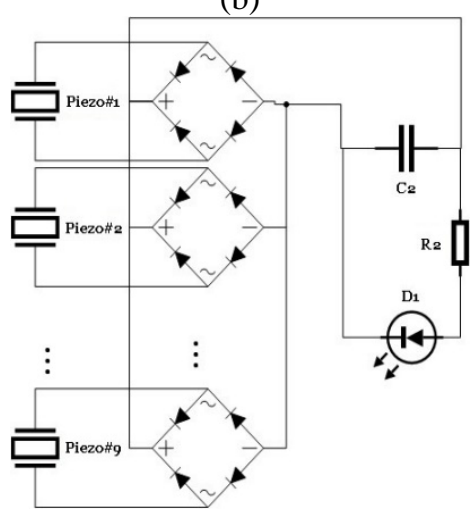

Fig. 8. (a) Test setup, (b) schematic of electrical circuit 
As shown below in Fig. 9, applying imprison load similar the walking load can turn on the eighteen LEDs.

(a)

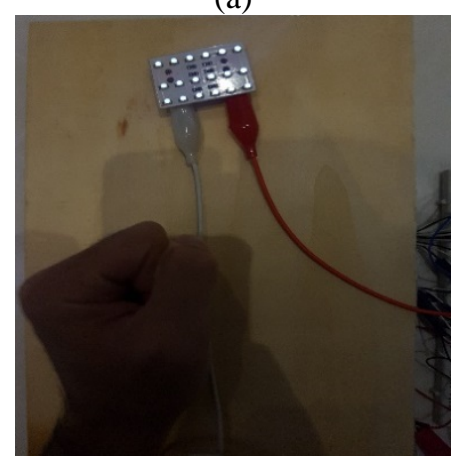

(b)

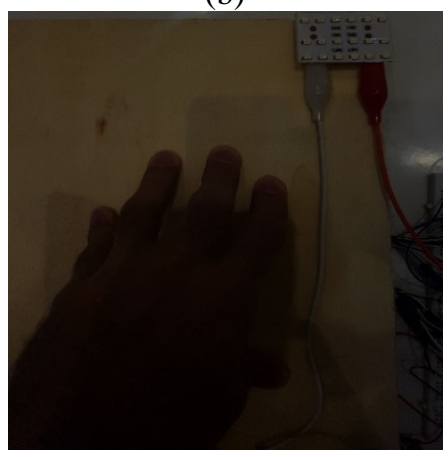

Fig. 9. LED lights on \& off (a): turn on, (b): turn off

By applying the dynamic load to the tile, the capacity is charged which remove the LEDs voltage ripple and keep the light quality. The system parameters should be designed to meet some functional aspects such as LEDs start time and time period of lighting.

To investigate the effect of parameters, force excitation is applied to tile at $t=0 \mathrm{~s}$ and it is removed at $t=55 \mathrm{~s}$. Input and output voltages of LEDs are monitored which makes it possible to detect onloff times of LEDs. To analyze the effect of resistance value on the system response, LEDs voltages are plotted in Fig. 10 for three different values of $R_{s}$ where the capacity is set as $C_{s}=1 \mu \mathrm{F}$.

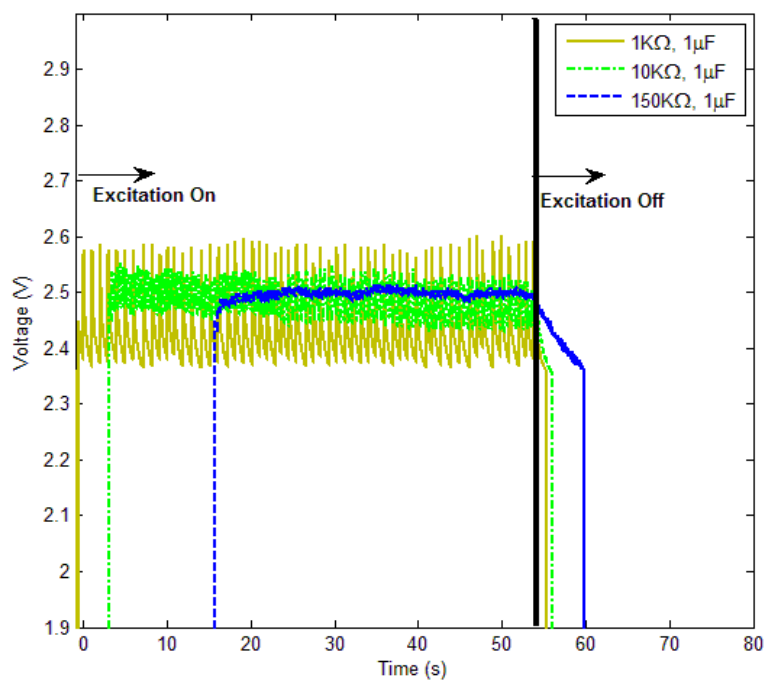

Fig. 10. Time response of LEDs voltage for $R_{s}=1 \mathrm{~K} \Omega, R_{s}=10 \mathrm{~K} \Omega, R_{s}=150 \mathrm{~K} \Omega$, and $C_{s}=1 \mu \mathrm{F}$

As seen in Fig. 10, because of low value of system time constant and fast discharge of capacitive, LEDs voltage oscillates with higher amplitude for lower values of resistance. This occurs by decreasing the applied force. On/off times of LEDs are depicted in Fig. 11.

On/off and lighting times of LEDs for $C_{s}=1 \mu \mathrm{F}$ and different values of $\mathrm{R}$ are presented in Table 2 . As seen in Fig. 11 and Table 2, despite the positive effect of increasing resistance on LEDs lighting time after removing external mechanical load, it increases the LEDs on time dramatically which leads to lower lighting time.

To overcome this drawback, the $C_{s}=100 \mu \mathrm{F}$ is replaced with previous one. LEDs voltages are plotted in Fig. 12 for three different values of $R$ where the capacity is set as $C_{s}=100 \mu \mathrm{F}$ and the important characteristics of system are presented in Table 3.

It is clear that for $C_{s}=100 \mu \mathrm{F}$ and $R_{s}=150 \mathrm{~K} \Omega$ the lighting time of LEDs increase to 100s and the LED voltages has lowest ripple. 


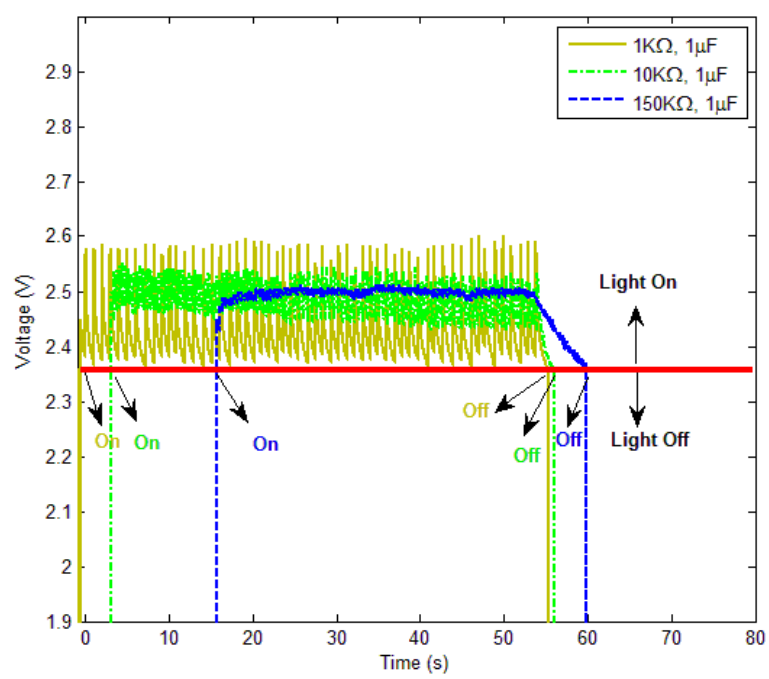

Fig. 11. On/OFF times of LEDs for $R_{s}=1 \mathrm{~K} \Omega, R_{s}=10 \mathrm{~K} \Omega, R_{s}=150 \mathrm{~K} \Omega$, and $C_{s}=1 \mu \mathrm{F}$

Table 2. On/off and lighting times of LEDs for $C_{s}=1 \mu \mathrm{F}$ and different values of $R_{S}$

\begin{tabular}{cccc}
\hline & On Time & Off Time & Lighting Time \\
\hline$R_{s}=1 \mathrm{~K} \Omega$ & 0.5 & 55.1 & 54.6 \\
$R_{s}=10 \mathrm{~K} \Omega$ & 4.3 & 55.9 & 51.6 \\
$R_{S}=150 \mathrm{~K} \Omega$ & 15.2 & 59.7 & 44.5 \\
\hline
\end{tabular}

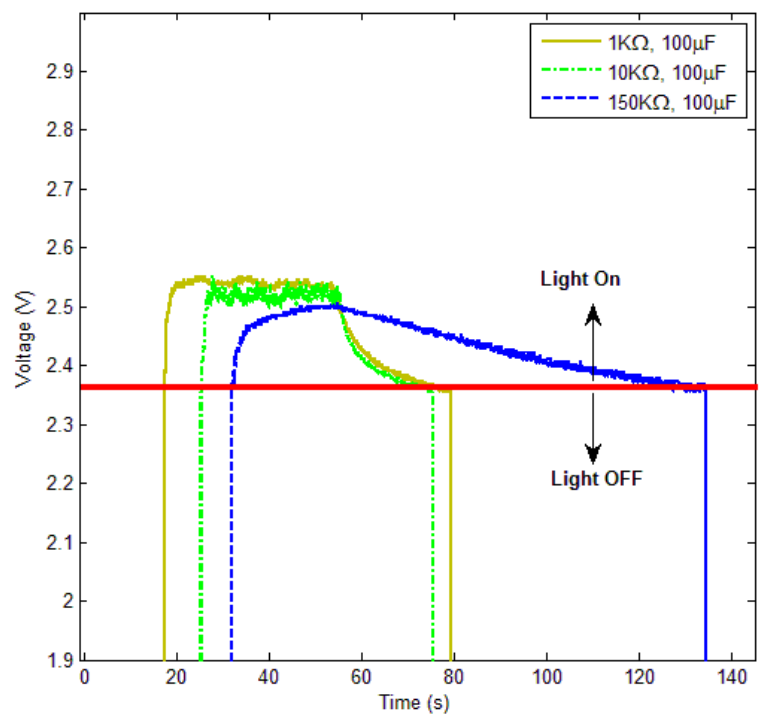

Fig. 12. Time response of LEDs voltage for $R_{s}=1 \mathrm{~K} \Omega, R_{s}=10 \mathrm{~K} \Omega, R_{s}=150 \mathrm{~K} \Omega$, and $C_{s}=100 \mu \mathrm{F}$

Table 3. On/off and lighting times of LEDs for $C_{s}=100 \mu \mathrm{F}$ and different values of $R_{S}$

\begin{tabular}{cccc}
\hline & On Time & Off Time & Lighting Time \\
\hline$R=1 \mathrm{~K} \Omega$ & 17.6 & 75.5 & 57.9 \\
$R=10 \mathrm{~K} \Omega$ & 25.5 & 79.5 & 54.0 \\
$R=150 \mathrm{~K} \Omega$ & 32.3 & 133.3 & 101.0 \\
\hline
\end{tabular}

\section{Conclusion}

design of piezoelectric tile for energy harvesting was presented in this paper. Two tiles with different mechanical designs were fabricated and compared with together. It was shown that titling the piezoelectric disks 
in tile leads to higher mechanical stress and respectively higher generated voltage and electrical power. Moreover, the tile is examined for lightening aim and it is shown that by proper selecting of electrical circuit elements results in higher lightening time after removing excitation load and keeps the light on for higher time period. The tile with proposed mechanical and electrical design can be useful at wide area and different applications.

\section{References}

[1] Kang MG, Jung WS, Kang CY, Yoon SJ. Recent progress on PZT based piezoelectric energy harvesting technologies. Actuators. 2016;5(1).

[2] Niu X, Yu J, Wang S. Experimental study on low-temperature waste heat thermoelectric generator. J Power Sources. 2009;188(2):621-626.

[3] Ahmad S, George C, Beesley DJ, Baumberg JJ, De Volder M. Photo-Rechargeable Organo-Halide Perovskite Batteries. Nano Lett. 2018;18(3):1856-1862.

[4] Zi Y, Lin L, Wang J, Wang S, Chen J, Fan X, et al. Triboelectric-pyroelectric-piezoelectric hybrid cell for high-efficiency energy-harvesting and self-powered sensing. Adv Mater. 2015;27(14):2340-2347.

[5] Bhatia D, Lee J, Hwang HJ, Baik JM, Kim S, Choi D. Energy Harvesting: Design of Mechanical Frequency Regulator for Predictable Uniform Power from Triboelectric Nanogenerators. Adv Energy Mater. 2018;8(15):1870072.

[6] Thainiramit P, Yingyong P, Isarakorn D. Impact-driven energy harvesting: Piezoelectric versus triboelectric energy harvesters. Sensors (Switzerland). 2020;20(20):1-20.

[7] Chen X, Xu S, Yao N, Shi Y. 1.6 v nanogenerator for mechanical energy harvesting using PZT nanofibers. Nano Lett. 2010;10(6):2133-2137.

[8] Tong C, Tong C. Emerging Materials for Energy Harvesting. Introduction to Materials for Advanced Energy Systems. 2019. 719-817.

[9] Ottman GK, Hofmann HF, Bhatt AC, Lesieutre GA. Adaptive piezoelectric energy harvesting circuit for wireless remote power supply. IEEE Trans Power Electron. 2002;17(5):669-676.

[10] Safaei M, Sodano HA, Anton SR. A review of energy harvesting using piezoelectric materials: State-of-theart a decade later (2008-2018). Smart Mater Struct. 2019;28(11).

[11] Puscasu O, Counsell N, Herfatmanesh MR, Peace R, Patsavellas J, Day R. Powering Lights with Piezoelectric Energy-Harvesting Floors. Energy Technol. 2018;6(5):906-916.

[12] Hwang W, Kim KB, Cho JY, Yang CH, Kim JH, Song GJ, et al. Watts-level road-compatible piezoelectric energy harvester for a self-powered temperature monitoring system on an actual roadway. Appl Energy. 2019;243:313-320.

[13] Abadi PB, Darlis D, Suraatmadja MS. Green energy harvesting from human footsteps. MATEC Web Conf. 2018;197:6-9.

(C) 2021 by the author(s). This work is licensed under a Creative Commons Attribution 4.0 International License (http://creativecommons.org/licenses/by/4.0/). Authors retain copyright of their work, with first publication rights granted to Tech Reviews Ltd. 